Itinéraires Itinéraires

Littérature, textes, cultures

\title{
Homographèse : identité corporelle et différence sexuelle
}

Lee Edelman

Traducteur : Pierre Zoberman

\section{OpenEdition \\ Journals}

Édition électronique

URL : http://journals.openedition.org/itineraires/375

DOI : 10.4000/itineraires.375

ISSN : 2427-920X

Éditeur

Pléiade

\section{Édition imprimée}

Date de publication : 1 mai 2009

Pagination : 133-160

ISBN : 978-2-296-08444-5

ISSN : $2100-1340$

\section{Référence électronique}

Lee Edelman, « Homographèse : identité corporelle et différence sexuelle », Itinéraires [En ligne], 2009-1 | 2009, mis en ligne le 03 septembre 2014, consulté le 01 juillet 2020. URL : http:// journals.openedition.org/itineraires/375; DOI : https://doi.org/10.4000/itineraires.375

Ce document a été généré automatiquement le 1 juillet 2020

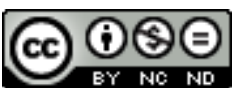

Itinéraires est mis à disposition selon les termes de la licence Creative Commons Attribution - Pas d'Utilisation Commerciale - Pas de Modification 4.0 International. 


\title{
Homographèse : identité corporelle et différence sexuelle
}

\author{
Lee Edelman \\ Traduction : Pierre Zoberman
}

\section{NOTE DE L'ÉDITEUR}

Ce texte a été publié sous le titre « Homographesis » dans Homographesis: Essays in Gay Literary and Cultural Theory de Lee Edelman (New York : Routledge, 1994). Il a été traduit de l'américain par Pierre Zoberman.

1 Au printemps 1987, lorsqu'on m'a invité à prendre part à un colloque pour inaugurer le Centre d'études lesbiennes et gay de Yale, les organisateurs m'ont demandé de participer, avec d'autres chercheurs gay, à un panel dont le titre posait avec insistance la question de l'identité : «En quoi la littérature gay est-elle gay ? En quoi la littérature lesbienne est-elle lesbienne?» Bien que l'affichage de notre séance eût changé substantiellement quand le programme fut finalement diffusé, le titre avait conservé, de manière clairement concertée - et presque agressive - la forme interrogative ; mais la question soulevée était maintenant plus tranchante, et plus sceptique : «Peut-il y avoir une critique gay?» Toutes ces questions présupposent implicitement qu'en tant que critiques littéraires gay, nous appliquons, ou à tout le moins nous devrions appliquer tout notre intérêt et toute notre énergie à établir la spécificité d'une méthodologie critique gay ou lesbienne. Ces questions semblaient en effet appeler ceux d'entre nous qui travaillent dans des perspectives lesbiennes, gay, bisexuelles, "queer", non hétérosexuelles ou anti-homophobes, non seulement à prendre explicitement en compte la sexualité dans les textes à propos desquels nous écrivons, mais encore à rendre lisible dans notre production critique une entreprise théorique spécifiquement gay. En somme, ces questions nous imposent d'être prêts à poser, voire à défendre l'existence d'une identité critique singulière, reconnaissable et, donc, reproductible; à réduire la critique lesbienne et gay à un produit de consommation courante en l'étiquetant comme parfum particulier de la théorie littéraire, susceptible 
de trouver sa part de marché propre dans l'économie de luxe de la production littéraire. Du même coup, ces questions nous demandaient de localiser «la différence homosexuelle" comme entité déterminée plutôt que comme relation différentielle instable et nous invitaient à donner à notre auditoire des lignes directrices pour définir «l'homosexuel» ou même «l'homosexualité». Comment, semblaient-elles nous demander, la critique littéraire peut-elle voir ou reconnaître «l'homosexuel » afin de placer l'homosexualité sous le regard de la théorie? Comment, en d'autres termes, "l'homosexualité " peut-elle trouver sa place dans le discours critique contemporain de manière à ne plus être sans marque, invisible, ou perceptible seulement lorsqu'elle se pavane dans le travesti [drag] thématique ou référentiel le plus flagrant?

\section{La lecture du corps homosexuel : une herméneutique du soupçon}

2 Cet impératif de mettre au jour «la différence homosexuelle » comme objet d'examen cognitif et perceptif reste naturellement au centre des préoccupations d'une politique de libération attachée à la nécessité sociale d'ouvrir, voire d'éliminer la porte du placard $^{2}$. Elle fait sien le désir de mettre en pleine lumière les différences historiques, politiques et représentationnelles inscrites dans les lectures que notre société fait de l'écart sexuel, et nous pousse à reconnaître la différence sexuelle là où elle réussit à passer inaperçue. Mais c'est précisément là que le projet libérationniste peut aisément faire écho, quoique dans une tonalité différente, à l'homophobie, qui n'a de cesse de présenter la codification et l'enregistrement des identités sexuelles comme un enjeu social de première importance. Bien qu'ils cherchent à mettre en œuvre des ordres du jour radicalement différent, le défenseur des gays et l'agent responsable de l'application des normes homophobes investissent tous deux la question de la lisibilité gay d'un caractère pressant et douloureux - lequel caractère atteste, au moins en partie - leur anxiété spécifique respective et ce qui est en jeu, de manière distincte, pour chacun, dans la lecture que la culture fait de l'homosexualité et sa capacité de reconnaître comme homosexuel tout individu donné. Des pratiques comme le " outing " (révélation publique de l'orientation sexuelle de lesbiennes ou d'hommes gay et particulièrement de ceux qui ont accès à l'autorité culturelle et en profitent pour perpétuer la stigmatisation de l'homosexualité) naissent, bien sûr, du fait que l'homosexualité reste, pour la plupart, illisible dans la personne des gays et des lesbiennes qu'ils rencontrent, au travail, dans leur famille, dans leur gouvernement, à la télévision ou au cinéma. Tout comme le outing permet de rendre visible une dimension de la réalité sociale que les présupposés d'une idéologie hétérosexiste bloquent de fait complètement, cette même idéologie n'a cessé d'affirmer, tout au long $\mathrm{du} \mathrm{xx}^{\mathrm{e}}$ siècle, la nécessité de «lire» le corps en tant que signifiant de l'orientation sexuelle. L'hétérosexualité a ainsi pu renforcer son statut d'autorité comme " naturelle " (c'est-à-dire non marquée, authentique et non représentationnelle) en définissant le corps straight (c'est-à-dire hétérosexuel) par opposition à la "menace " d'une homosexualité " contre-nature »- une menace d'autant plus facile à évoquer en mobilisant les craintes que suscite la capacité déconcertante (et stratégiquement manipulable) qu'a l'homosexualité de "passer ", de rester invisible, pour faire surgir toute une variété de "savoirs" disciplinaires au moyen desquels elle pourrait être 
reconnue, démasquée, et, en dernière analyse, rendue à nouveau à une invisibilité d'encore plus mauvais augure.

Que ce genre de déchiffrage d'une homosexualité lisible, voire la simple éventualité d'un tel déchiffrage occasionne une anxiété sociale si puissante et une agression psychique si répandue est l'indice de l'importance critique, et de la valeur symptomatique, que notre culture en est venue à accorder à l'identification de «l'homosexuel »; et cela souligne, dans le même mouvement, la relation historique qui a produit la sexualité gay au sein d'un discours qui l'associe avec des figures de nomination ou d'inscription. En 1986 encore, le juge Warren F.Burger, dans un commentaire de soutien à l'opinion majoritaire, déposée dans le cadre de l'affaire Bowers contre Hardwick, est allé jusqu'à rappeler à la cour que "Blackstone avait caractérisé le "crime infâme contre-nature" comme un crime d'une "malignité plus profonde" que le viol, comme un acte odieux "dont la seule mention est une honte pour la nature humaine ${ }^{3}$ " $($ (Burger 1986 : 13) Blackstone était si conscient de l'impropriété inhérente à « la seule mention » de ce crime qu'il continue, dans un passage que Burger ne cite pas, en reconnaissant l'interdit de nommer qui finit par donner son nom au crime lui-même : "Il sera préférable d'imiter à cet égard la délicatesse de notre droit anglais, qui le traite, jusque dans ses formules d'inculpation, en crime indigne d'être nommé : "peccatum illud horribile, inter christianos non nominandum" (Blackstone 1899 : $4:$ 1377) Dans son histoire du droit criminel britannique, Sir Leon Radzinowicz suggère qu'une inquiétude analogue à l'égard de la relation subversive entre pratique homosexuelle et propriété linguistique a pu influer sur le rapport des membres de la Commission de droit criminel lorsque, en 1836, ils se mirent en devoir de recommander une réforme de la désignation légale des crimes capitaux.

Pour la sodomie, qu'ils évoquaient par l'expression «un crime sans nom d'une grande énormité ", ils l'excluaient pour lors de leur examen, peut-être avec le même sentiment que celui sous l'influence duquel Livingstone omit purement et simplement de le faire figurer dans le code pénal de la Louisiane, de peur que sa définition même "n'inflige une blessure durable à la moralité du peuple.» (Radzinowicz $1968: 316$ )

Si les pratiques homosexuelles ont été situées dans un rapport si puissant, et si puissamment proscripteur, au langage, les homosexuels, quant à eux, ont été vus comme produisant - et, chez certains «experts» dans le domaine médical, comme étant produits par - des corps porteurs d'un code anatomique distinct, et donc lisible. Dès 1750, John Cleland pouvait, dans ses Mémoires de Fanny Hill femme de plaisir, faire affirmer à Fanny Hill par Mrs. Cole, à propos du désir homoérotique masculin, que, " quelque effet que cette passion infâme ait pu avoir à d'autres époques et dans d'autres pays, cela semblait être une bénédiction propre à notre air et notre climat qu'il y eût une marque de peste imprimée de manière visible sur tous ceux qui en sont souillés, au moins dans cette nation ${ }^{4} »$. Au siècle suivant, tant Cesare Lombroso qu'Ambroise Tardieu, en suivant une logique qui n'était pas complètement différente, devaient affirmer avoir mis au point des profils physiologiques qui rendaient possible l'identification des "déviants sexuels", permettant ainsi à la médicalisation du discours sexuel, caractéristique du xix ${ }^{e}$ siècle, de servir plus efficacement les fins de la criminologie et du droit. Du coup, John Addington Symonds allait être en mesure d'invoquer l'idée reçue de l'homosexuel comme homme "aux désirs écrits sur la figure" (cité dans Weeks 1981: 111); et le narrateur de Teleny (1893), offrant une formule présentant une ressemblance frappante avec la précédente, pourrait exprimer 
une inquiétude très réelle à la pensée que sa sexualité hors la loi pût être marquée sur sa chair : "Comme Caïn, il me semblait que je portais mon crime écrit sur mon front. » (Wilde 1996: 175) Lorsqu'on arrive à la deuxième décennie $\mathrm{du} \mathrm{xx}^{\mathrm{e}}$ siècle, on est beaucoup moins prêts à accepter les notions de ce genre comme vérités scientifiques, mais elles restent disponibles pour une appropriation sous forme de métaphores, capables de renforcer efficacement la construction idéologique de la différence homosexuelle. Ainsi, Lord Summer pouvait affirmer en 1918 que les sodomites portaient «le poinçon d'une classe spécialisée et extraordinaire, tout autant que s'ils avaient porté sur le corps des particularités physiques»(dans Weeks 1981: 100). Autrement dit, les homosexuels étaient non seulement conçus en termes de relation radicalement puissante, quoique négative, aux pratiques signifiantes, mais en outre soumis à un impératif culturel qui les considérait comme textuels en soi - comme des corps qui pourraient bien porter un «poinçon » qu'on pouvait, et qu'on devait lire. De fait, dans de ce qui constitue l'une des formulations les plus explicites de cette perception du corps gay comme texte, Proust a remarqué la manière dont «sur la surface lisse d'un individu que rien ne distingue des autres, apparaît soudain, tracés avec une encre jusque-là invisible, les caractères qui composent le mot cher aux anciens Grecs » (Proust 1954 : 613-614). Que ce topos continue d'exprimer de manière effective le besoin de voir le corps gay comme lisible (besoin qui ne cesse de se manifester en entraînant des conséquences disciplinaires non négligeables), apparaît clairement dans sa réarticulation quelque quarante ans plus tard dans Another Country de James Baldwin: "Comment Eric aurait-il pu savoir que ses visions, aussi indéchiffrables qu'elles fussent pour lui, étaient inscrites dans chacun de ses gestes, trahies par la moindre inflexion de voix, et vivaient dans ses yeux avec tout l'éclat, la beauté et la terreur du désir?» (Baldwin 1964 : 114) L'importance ainsi attribuée à l'homosexualité est massivement surdéterminée. Bien que l'homosexualité ait été désignée comme un crime indigne d'être nommé chez des chrétiens, et bien qu'elle ait depuis longtemps été comprise, et représentée, comme " amour qui n'ose pas dire son nom ", la culture judéo-chrétienne a avidement cherché à lire une vaste gamme de signifiants comme autant de signes de ce que nous définissons aujourd'hui par « désir homosexuel ». Alan Bray a publié d'utiles réflexions sur la transition historique en Grande-Bretagne "de l'homosexualité diffuse du xvII siècle", homosexualité qui trouvait son potentiel signifiant dans une association mythique avec les sorciers et les hérétiques, les loups-garous et les basilics, à l'émergence au siècle suivant "d'une culture qui prend la suite [...] dans laquelle l'homosexualité pouvait être exprimée, et donc reconnue : les vêtements, les gestes, le langage, des bâtiments particuliers et des lieux publics particuliers - autant de choses que l'on pouvait identifier comme porteuses de connotations spécifiquement homosexuelles5.» (Bray 1982 : 92) Avec cette transition, nous entrons dans une ère dans laquelle l'homosexualité est finalement constituée socialement sous des formes qui, non seulement la rendent disponible à la signification, mais encore lui cèdent le pouvoir de signifier l'instabilité de la fonction signifiante en elle-même, la nature arbitraire et ténue de la relation entre tout signifiant et son signifié. Elle en arrive à figurer (et à être figurée en termes de) la subversion de l'ordre théologique à travers l'hérésie, de l'ordre politique à travers la trahison et de l'ordre social à travers le dérangement des rôles et des stéréotypes codifiés des genres sexués (genders). Aussitôt que l'homosexualité peut être localisée et, par conséquent, être lue au sein du paysage social, elle devient sujette à une dispersion métonymique qui permet de l'apercevoir pratiquement partout. Le domaine de la 
sexualité - qui est toujours, toujours, sous le régime du patriarcat, impliqué dans le domaine des relations de pouvoir et produit par lui, bien qu'en aucune façon identique à lui - n'est pas seulement scindé par la conscience de possibilités homosexuelles; il n'est pas seulement divisé entre les sphères distinctes, mais inégales des relations sexuelles homo- ou hétérosexuelles. Non: l'homosexualité en arrive à signifier la perméabilité potentielle de tout signifiant sexuel - et, par extension, de tout signifiant comme tel, à une signification " autre ». Dès que la sexualité peut être lue à la lumière de l'homosexualité, toute sexualité est sujette à une herméneutique du soupçon.

Or, si l'entreprise culturelle de lecture de l'homosexualité réclame que l'on affirme que l'homosexuel est marqué de manière distinctive et lisible, elle doit aussi reconnaitre que ces marquages ont été, peuvent être ou peuvent passer inaperçus et imperceptibles. On trouve une conséquence historiquement spécifique de cette conscience potentiellement déstabilisatrice dans l'implication réciproque entre homophobie et paranoïa dont Eve Kosofsky Sedgwick trace brillamment les contours :

C'est l'acharnement paranoïaque déployé par les non-homosexuels de ce siècle à élever des barrières définitionnelles entre "l'homosexuel» (la minorité) et «l'hétérosexuel " (la majorité), et surtout par des hommes à l'encontre d'autres hommes, qui mine le plus toute possibilité de croire en "l'homosexuel » comme catégorie de personne non problématiquement distincte ${ }^{6}$. (Sedgwick 1988 : 55)

Comme Sedgwick le note ailleurs, ces "barrières définitionnelles" sont les sites, instaurés comme mesure défensive, d'une volonté brutalement anxieuse de puissance à l'égard de l'interprétation de la subjectivité [selfhood] (paradigmatiquement masculine sous un régime social de structure patriarcale), une volonté de puissance qui « met en œuvre aussi la structure d'un projet érotique/érotophobique beaucoup plus spécifique : le projet de la paranoïa. Comme le dit l'expression de connivence la plus pure, « il faut en être pour le reconnaître ${ }^{7} »$. L'accès interprétatif au code qui rend lisible l'homosexualité porte en soi le stigmate d'une relation trop intime au code et à la mécanique de sa production, ce qui risque de situer le lecteur trop au fait des signes de l'homosexualité dans le contexte de ce que Sedgwick nomme "une terrible reconnaissance-miroir projective" (Sedgwick 1986: 107). Même s'il peut ainsi être aussi dangereux de lire que de ne pas savoir lire l'homosexualité, elle conserve dans les deux cas une relation déterminante à la textualité et à la lisibilité des signes.

\section{Homosexualité et métaphore : I'homographèse comme essentialisation}

7 Ce qui garantit toutes ces versions d'une inscription graphique de l'homosexualité et qui rend possible la culture de paranoïa dont Sedgwick dresse si brillamment l'anatomie, c'est, comme Michel Foucault l'affirme dans son Histoire de la sexualité, une transformation des pratiques discursives qui gouvernent l'articulation moderne de la sexualité elle-même. Remarquant que la sodomie constituait une des catégories des " actes interdits » des « anciens codes civil ou canonique », Foucault pose que

l'homosexuel du XIX ${ }^{\mathrm{e}}$ siècle est devenu un personnage, un passé, une histoire et une enfance, un caractère, une forme de vie ; une morphologie aussi, avec une anatomie indiscrète et peut-être une physiologie mystérieuse. Rien de ce qu'il est au total n'échappe à sa sexualité. Partout en lui, elle est présente : sous-jacente à toutes ses conduites, parce qu'elle en est le principe insidieux et indéfiniment actif : inscrite sans pudeur sur son corps et son visage parce qu'elle est un secret qui se trahit 
toujours. Elle lui était consubstantielle, moins comme un péché d'habitude que

comme une nature singulière. (Foucault 1976 : 59)

L'homosexualité ne devient ce qui est « écrit de manière impudique » sur l'« anatomie indiscrète » d'un corps spécifiquement homosexuel qu'à partir du moment où elle cesse d'être considérée comme un ensemble d'actes ou de comportements universellement disponibles, dont aucun n'a de relation privilégiée avec l'identité « sexuelle » du sujet, pour devenir au contraire, dans la formulation de Foucault, « la racine de toutes [les] actions » et donc une caractéristique définitoire de l'agent, le sujet, avec lequel elle est maintenant vue comme « consubstantielle».

Une manière de reformuler ce glissement discursif est de le voir comme une transformation du cadre rhétorique ou tropologique dans le contexte duquel le concept de «sexualité » lui-même est produit : la transformation d'une lecture de la relation du sujet à la sexualité comme contingente ou métonymique en une lecture dans laquelle la sexualité est réinterprétée comme essentielle ou métaphorique. Lorsque l'homosexualité n'est plus entendue comme un ensemble d'actes distincts, mais comme une " anatomie indiscrète ", nous sommes en présence d'un puissant impératif d'ordre tropologique, celui de produire un emblème ou une métaphore visible pour marquer la "nature singulière» qui maintenant définit ou identifie un type spécifiquement homosexuel. Mais cette marque ou emblème visible doit être reconnue comme figure de la conceptualisation, désormais métaphorique, de la sexualité même - une figure de la relation privilégiée à l'identité dont le sexuel sera désormais porteur. Donc, dans l'esprit de l'impératif anthropologique des sciences sociales $d u x x^{e}$ siècle, «l'homosexuel » a pu faire son apparition sur la scène culturelle par le biais de l'attribution d'une signification essentielle - c'est-à-dire l'attribution d'une puissance métaphorique - à certains détails anatomiques fortuits qui seraient, pour l'observateur averti, aussi indiscrets et révélateurs à l'égard de la "vérité » sur l'« identité sexuelle » d'une personne que les rêves ou les symptômes somatiques devaient l'être à l'égard de la « vérité » de l'inconscient pour le champ émergent de la psychanalyse.

9 Ainsi, la sexualité, dans l'acception actuelle du terme pour désigner une organisation et une orientation systématiques du désir, commence d'exister quand le désir - que Lacan, déployant les implications des affirmations de Freud avant lui, définit explicitement comme une métonymie - est faussement reconnu et faussement interprété comme métaphore ${ }^{8}$. Pourtant, si nous voyons cette fausse interprétation comme une " erreur ", c'est une erreur qui est inséparable de la sexualité telle que nous la connaissons, puisqu'on ne peut identifier la sexualité au métonymique sans reconnaître que l'acte même d'identification par lequel elle est constituée en tant que sexualité revient déjà à poser son sens en termes de cohérence et de nécessité métaphoriques. Autrement dit, on doit reconnaître du même coup que la métonymie elle-même peut seulement produire du «sens » dans le contexte d'une tradition logocentrique qui privilégie la métaphore comme nom de la relation d'essence, la relation paradigmatique, qui investit le langage de "sens » à travers la référence à un signifié imaginé comme quelque part présent à soi. Comme Lacan l'écrit dans un contexte différent, «la métonymie est là dès le début et c'est elle qui rend la métaphore possible ${ }^{9}$ »; mais ce n'est que dans la logique de la métaphore que la métonymie en tant que telle peut être "identifiée » et reconnue rétroactivement comme ayant "été " là depuis le commencement. C'est-à-dire que la métaphore solidarise les glissements arbitraires caractéristiques de la métonymie en unités de "sens" qui sont perçues comme identités ou présences représentationnelles. Ainsi l'histoire a fait que la sexualité a été 
investie d'une valeur métaphorique plutôt que métonymique, ce qui a rendu possible la recherche de signifiants qui témoigneraient de la présence de cette identité sexuelle ou « essence » nouvellement posée. Et Arno Karlen peut ainsi renforcer l'affirmation de Foucault et montrer une fois de plus la convergence des discours médical et juridique du XIX ${ }^{e}$ siècle sur l'intérêt porté à la question de la taxinomie sexuelle, en notant que les « deux auteurs les plus largement cités » sur l'homosexualité «après le milieu du siècle furent les experts médico-légaux en Allemagne et en France, les docteurs Casper et Tardieu. Tous deux se préoccupaient surtout de savoir si la race dégoûtante des pédérastes pouvait être identifiée physiquement pour les tribunaux" (Karlen 1971: 185).

10 En citant ces documents, je veux attirer l'attention sur la formation d'une catégorie, "la personne homosexuelle", dont la condition de possibilité même est sa relation à l'écriture ou la textualité, et en particulier la manière dont elle articule une différence «sexuelle» interne à l'identité masculine qui engendre la nécessité de lire certains corps comme visiblement homosexuels. L'inscription de "l'homosexuel» dans une tropologie qui le produit dans une relation déterminante à la notion même d'inscription est la première chose que j'entends dénoter par l'emploi du terme "homographèse $»^{10}$. Ce néologisme par lequel je souhaite désigner un réseau de préoccupations au cœur de toute discussion théorique de l'homosexualité en rapport avec et comme produit de l'écriture ou de la textualité, incorpore littéralement dans sa structure-et incorpore figurativement en renvoyant au corps-la notion de « graphèse » [graphesis], abordée dans un numéro de Yale French Studies édité par MarieRose Logan. Dans son introduction à ce numéro, Logan définit graphesis comme «le point nodal de l'articulation d'un texte " qui "dé-limite le lieu où la question de l'écriture se pose » et "décrit l'action d'écrire dans son actualisation dans le texte, indépendamment de la notion d'intentionnalité »(Logan 1975 : 12). Autrement dit, à la suite de la caractérisation post-saussurienne de l'écriture par Derrida comme un système de "différance » qui opère sans termes positifs et diffère indéfiniment l'accomplissement de l'identité comme présence à soi, la "graphèse ", l'entrée dans l'écriture que l'«homographèse » voudrait spécifier n'est pas seulement celle dans laquelle "l'identité homosexuelle » est conceptualisée de manière différentielle par une culture homosexuelle comme quelque chose d'écrit lisiblement sur le corps, mais aussi celle dans laquelle le sens même d'« identité sexuelle » est déterminé par le biais de son assimilation à la position de l'écriture au sein de la tradition de la métaphysique occidentale. Ma thèse sera que l'«écriture" à laquelle l'homosexualité a historiquement été assimilée désigne la réduction de la « différance » à une question de différence déterminée ; à partir de la position de la culture dominante elle assigne à l'homosexualité une forme de représentation secondaire, stérile et parasitaire qui se situe dans un rapport à l'identité hétérosexuelle analogue à celui que l'écriture entretient dans la métaphysique phonocentrique dont Derrida retrace la continuité à travers la philosophie occidentale de Platon à Freud (et au-delà), avec la parole ou la voix. Cependant, en tant que principe d'articulation différentielle, l'écriture, spécialement quand on l'entend comme une approximation de ce processus signifié par "graphèse ", ne fonctionne comme articulation de l'identité qu'en relation avec des signes qui sont structurés, selon la formule de Jacques Derrida, par leur «non-identité à soi ${ }^{11} »$. Ainsi, bien que l'écriture marque ou décrive les différences dont dépend la spécification de l'identité, elle a aussi pour effet, comme l'écrit Logan, de « dé(s-é)crire [de-scribe]», d'effacer ou de défaire l'identité en posant la différence comme fausse 
reconnaissance d'une "différance », dont la négativité, l'articulation purement relationnelle met en question la possibilité de toute présence positive ou de toute identité discrète. Comme l'écriture, donc, le terme d'homographèse vise à désigner deux opérations à la fois : l'une, au service des fins d'un ordre social conservateur attaché à la codification des identités dans son effort d'inscription disciplinaire; l'autre, en résistance à cette catégorisation, n'ayant de cesse de dé-crire les identités que l'ordre a inscrites de manière si oppressive. Le postulat que ces deux opérations, orientées de la sorte dans deux directions opposées, habitent un signifiant unique, ne peut que créer un certain degré de confusion, mais la confusion qui naît lorsque la différence se replie en identité et l'identité se déploie en différance est, comme je le suggérerai dans ce qui suit, centrale à la problématique de l'homographèse. Car, et ici j'anticipe un peu, si la production culturelle de l'identité homosexuelle en termes d'une "anatomie indiscrète » exerce un contrôle sur le sujet (qu'il soit straight ou gay), en soumettant son autoreprésentation corporelle à un examen analytique, le caractère arbitraire des indices qui permettent d'identifier la "sexualité »-ce qui revient à dire l'homosexualité - atteste l'impératif culturel de produire, à des fins de régulation idéologique, une différence putative au sein du groupe de corps masculins qui passeraient, sans cela, pour «les mêmes", si «l'identité sexuelle » n'était pas désormais interprétée comme une essence installée dans l'espace instable entre le «sexe » et la catégorie récemment articulée de «sexualité » ou d'« orientation sexuelle».

11 Pour rendre aussi claires que possibles les implications de l'homographèse, je donnerai le détail des manières dont elle désigne, d'un côté, une pratique normalisante de discrimination culturelle (qui génère, en réponse, l'autonomination qui finit par déboucher sur une politique d'affirmation de soi pour une communauté gay qui accède au statut de minorité) et, de l'autre, une résistance stratégique à la réification de la différence sexuelle. Dans le premier sens, l'homographèse renverrait au mécanisme culturel par lequel l'écriture est mise en relation avec la question de la différence sexuelle pour concevoir le corps gay comme un texte, entraînant par là une intervention de grande envergure dans la régulation et la police des identités sexuelles. Le processus qui construit l'homosexualité en objet de discours, en catégorie culturelle à propos de laquelle on peut penser, parler ou écrire, coïncide, dans cette logique d'homographèse, avec celui par lequel le sujet homosexuel est représenté comme habitant, ou même plutôt comme étant un corps qui exige constamment d'être lu, un corps sur lequel sa sexualité est toujours déjà inscrite.

12 Tout comme la surimposition d'une valeur métaphorique réputée stable sur la catégorie métonymique du désir rend possibles des figurations conventionnelles de la lisibilité d'une « morphologie » homosexuelle distinctive, elle produit le besoin d'établir un tel emblème de la différence homosexuelle, qui situera solidement cette différence dans le registre de la visibilité. Cette référence à un analogue visible de la différence peut naturellement prendre appui sur les associations culturelles qui ont uni la sodomie à l'efféminement dans la pensée européenne longtemps avant l'« invention » de l'homosexuel (voir Trumbach 1977). Comme Randolph Trumbach l'a noté, entre le $\mathrm{XII}^{\mathrm{e}}$ et le $\mathrm{XVIII}^{\mathrm{e}}$ siècle, les hommes qui pratiquaient la sodomie avec d'autres hommes avaient déjà toutes les chances d'être caractérisés comme efféminés, mais, dans la mesure où les relations entre hommes n'étaient pas vues comme l'expression d'une " orientation" sexuelle, ces associations avec l'efféminement étaient largement métonymiques, et trouvaient leur point d'ancrage dans des aspects du comportement 
définis en termes d'affectation ou d'imitation ${ }^{12}$. Trumbach enchaîne sur la suggestion qu'il est

très probable que, dans le Londres du début du xvII ${ }^{e}$ siècle, il y avait un réseau ou une sous-culture sodomitique qui, parce qu'ils n'étaient pas aussi développés qu'ils le deviendraient par la suite, parce que la régulation sociale n'était pas encore aussi efficace que les Sociétés pour la réforme des mœurs allaient la rendre et surtout parce que la sodomie entre hommes n'était pas encore conçue comme excluant les relations sexuelles avec des femmes, n'étaient peut-être pas au xvII siècle la cible du type d'attaques qu'ils subissaient occasionnellement au XvIII'. (Trumbach 1987 : 119)

13 Dans la transformation discursive dont les travaux de Foucault nous donnent une idée, ces connexions contingentes entre sodomie et efféminement subissent un glissement et deviennent des équivalences essentielles ou métaphoriques dès que la sexualité ellemême subit une totalisation métaphorisante en catégorie ou en essence, en identité fixée et exclusive. Selon Trumbach :

Dans cette culture, le sodomite devient un individu qui s'intéresse exclusivement à ceux qui sont du même genre sexué [gender] et d'une passivité et d'un efféminement invétérés. Un homme qui s'intéresse aux femmes ne courait jamais le risque de devenir efféminé comme cela avait été le cas jadis, puisqu'il n'y avait aucune chance qu'il puisse se soumettre passivement à un autre mâle. Dans ce monde-là, ce n'était pas de la diffamation que de dire qu'un homme était un débauché, ou qu'il fréquentait les prostituées - c'était une preuve de sa masculinité - et ce genre de cause disparut des tribunaux, mais les hommes adultes ne pouvaient tolérer l'accusation d'être sodomites. (Trumbach $1987: 118$ )

14 À partir du moment où la sexualité devient si intimement liée à une idéologie définie par le binarisme des genres et à partir du moment où la sexualité masculine en particulier est susceptible d'être (mal) lue en relation avec des identités hétérosexuelle et homosexuelle radicalement discontinues, il devient à la fois possible et nécessaire de poser le marqueur de la «différence homosexuelle» en termes de représentation visuelle - dans les termes précisément que la psychanalyse définit comme jouant un rôle fondamental dans le processus par lequel les distinctions anatomiques s'inscrivent dans l'ordre symbolique de la sexualité et y prennent un sens. Cependant, contrairement à la différence des genres, dont beaucoup de féministes et de psychanalystes font le point d'ancrage de la notion même de différence, la « différence homosexuelle " produit l'impératif de la reconnaître et de la dévoiler dans la mesure précisément où elle menace de demeurer non marquée et non détectée et par là même de venir troubler la stabilité des paradigmes à travers lesquels la différence des genres peut être interprétée et imposée.

15 Ainsi, si l'homographèse réfère à l'acte par lequel l'homosexualité est mise en écriture sous l'égide de l'écriture même, elle suggère en même temps la mise en écriture - et donc l'entrée dans le domaine de la différance-du même, de la similitude ou des métaphores essentialisantes de l'identité (et spécifiquement de l'identité hétérosexuelle masculine comme figure exemplaire de l'autonomie et de la cohérence du sujet comme présent à lui-même) que l'homographèse, dans sa première acception, est censée assurer. En déconstruisant la logique binaire de la différence sexuelle sur laquelle l'identité symbolique est fondée, la graphèse, l'inscription culturelle des possibilités homosexuelles perturbe de manière effective la stabilité que la perception visuelle de la «ressemblance » et de la «différence» aurait autrement pour effet d'ancrer. Insistant sur un second ordre de différence sexuelle visuellement inscrite, 
l'homographèse répond, tout en la redoublant, à l'anxiété qui concerne la cohérence des identités pour la solidification desquelles elle a initialement été suscitée. Car la tropologie récurrente du corps gay inscrit indique, par son affirmation défensive d'un marqueur d'altérité sexuelle, une crainte que l'institutionnalisation catégorielle de la "différence homosexuelle» puisse mettre en question l'intégrité et la fiabilité de l'identité anatomique du même comme garante de l'identité sexuelle, que l'élaboration de la différence entre les catégories proliférantes du sexe, du genre et de la sexualité et à l'intérieur même de ces catégories puisse compromettre la certitude par laquelle on pourrait connaître sa propre identité. Pour dire les choses simplement, la position historique de la catégorie de "l'homosexuel» textualise l'identité masculine comme telle, la soumettant à l'exigence aliénante d'être « lue », et menaçant, par conséquent, de priver la «masculinité » de son statut privilégié de paradigme auto-authentifiant du naturel ou de ce qui va évidemment de soi. Maintenant, elle doit jouer [perform] cette évidence, représenter sa propre différence à la " masculinité » dérivative et artificielle de l'homme gay. L'homosexuel, dans un contexte social de ce type, est contraint à porter le stigmate de l'écriture ou de la textualité comme son identité, comme l'expression même de son anatomie par une culture masculiniste hautement soucieuse de préserver l'autorité de son identité par le biais de l'institution d'une homographèse dont la logique de lisibilité, de différence graphique, voudrait nier la « masculinité » commune, la relation signifiante commune à une « catégorie du mâle » des hommes gay aussi bien que des hommes straight.

\section{Dorian Gray et Charlus : I'inquiétant homographe}

Pour présenter les choses sous un autre angle, le travail disciplinaire de l'homographèse, dans sa première acception comme production d'identité, peut être déployé en marquage ou en articulation culturelle obligatoire de la lisibilité homosexuelle qui naît de la crainte que l'homosexuel ne soit inscrit dans ce que j'appellerais la sphère d'influence de l'homographe. En tant que structure explicitement graphémique, l'homographe fournit un point de référence utile pour l'examen d'une graphèse gay. Car, par homographe, on entend « un mot qui présente la même forme écrite qu'un autre mot, mais d'origine et de sens différents "; l'homographe pose donc la nécessité de lire la différence dans des graphèmes qui paraissent être les mêmes. Le Oxford English Dictionary, par exemple, cite une définition de 1873 qui décrit les homographes comme «identiques pour l'œil », et une autre qui renvoie « des groupes de mots identiques quant à l'orthographe, mais qui, en réalité, consistent peut-être en parties du discours différentes, ou même en mots qui n'ont aucun rapport ». Ainsi, bear comme signifiant qui désigne l'ours est étymologiquement distinct de bear comme signifiant pour désigner l'action de porter ou de supporter ; et, de la même manière, c'est seulement un accident métonymique de transformation linguistique qui a produit, à partir d'origines différentes, last, la forme qu'utilise un bottier et last, l'adjectif pour qualifier ce qui vient après tout le reste, «dernier ». Les homographes mettent l'accent sur la multiplicité des histoires qui informent les " identités " graphiques, qui conduisent ${ }^{13}$ à des situations où la qualité de "mêmeté ", auparavant soumise à la "graphèse " qui signifie l'écriture comme description ou comme désignation à travers la différenciation, révèle l'impossibilité de toute identité qui pourrait être présente en soi. La délimitation (et du coup la régulation) des identités que l'homographèse renforce cherche à affirmer une différence de "sens ", 
une différence au niveau "étymologique", entre l'être hétérosexuel et l'être homosexuel. Elle cherche à nier le fait qu'elle est impliquée dans l'ambiguïté sémantique de l'homographe en affirmant la présence, inscrite sur le corps gay, d'un analogue de la différence qui en fait une structure hétérographique, correspondant, de manière métaphorique, à l'hétérogénéité affirmée, à la différence essentielle, entre hétéro- et homo-sexualité.

Ainsi, ce n'est que dans sa seconde acception, comme mode de résistance stratégique ou analytique à la logique de l'identité comme régulation, que l'homographèse reconnaît, et même cherche et interroge sa relation à l'homographe, en soulignant à quel point l'homographe représente quelque chose de central à l'écriture ou "graphèse ", à laquelle l'homosexualité est liée par l'institutionnalisation de l'homographèse comme discipline de contrôle social. Après tout, l'homographe lui-même ne permet la spécification de ses significations diverses et sans lien entre elles que par le biais de son déploiement dans une structure grammaticale ou une chaîne syntagmatique particulière. Sans identité singulière, l'homographe (développant, en cela, une propriété de l'écriture - et, partant, du langage - en général) ne se sédimente en signification qu'en vertu de sa relation linéaire, sa relation métonymique à un contexte qui semble valider, c'est-à-dire "naturaliser ", une dénotation aux dépens d'une autre. Invoquant ainsi les collocations aléatoires de la métonymie pour mettre en question la correspondance que pose la métaphore entre essences ou qualités positives présentes en soi, le travail de l'homographèse (comme articulation de la logique de l'homographe) déconstruit l'homographèse (comme désignation du marquage d'une identité homosexuelle distincte et lisible). En dévoilant la non-coïncidence, ce qui paraît être le même, l'homographe, comme l'écriture, mine la sécurité de la distinction entre similitude et différence, et laisse apercevoir au passage le statut fictionnel du geste fondateur de la logique. En fait, si l'homosexualité tire et son nom et son identité culturelle de la mise en avant d'une relation du même au même entre le sujet et l'objet du désir, l'homographèse semble suggérer l'inévitabilité d'un échange de sens entre les préfixes homo et hétéro. L'impératif d'établir une différenciation catégorique entre une hétérosexualité et une homosexualité est au service du principe « hétérosexuel » d'une identité essentielle (et oppositive) tandis que l'homosexualité introduirait la différence ou l'hétérogénéité dans ce qui passe pour être le même. En d'autres termes, l'hétérosexualité cherche à assurer l'identité du même ou la pureté intérieure aux "opposés " catégoriques du «sexe » anatomique en affirmant sans cesse que les relations de désir doivent attester une différence imaginable seulement hors de ces deux catégories "naturelles", "allant de soi», et donc "entre» elles; et l'homosexualité, en revanche, multiplierait les différences que le désir peut appréhender par des voies qui menacent la cohérence interne des identités sexuées que l'ordre de l'hétérosexualité exige. L'homosexualité est donc constituée en catégorie pour désigner une condition qui doit être représentée comme déterminée, comme lisible, identifiable, dans la mesure précisément où elle menace de défaire la détermination de l'identité ; elle doit être métaphorisée en condition essentielle, en une orientation sexuelle, pour contenir le trouble qu'elle produit comme force de désorientation. En rappelant, dans ce contexte, la manière dont la métaphore fait appel à l'idée d'essence ou d'identité totalisable ${ }^{14}$, nous pouvons dire que l'homographèse, dans sa seconde acception, comme processus de déconstruction, démasque le lissement métonymique, la différence interne au "même » signifiant, que la métaphore aurait justement pour but de stabiliser ou de renier. Elle articule donc une différence par 
rapport à la différenciation binaire entre identité et différence, présence et absence, ces deux couples unis l'un à l'autre pour déterminer l'identité comme ressemblance et comme présence à soi. En ce sens, l'homographèse, dans un geste qui sauvegarde ce qu'il conteste, définit comme central à l'« homosexualité » un refus des spécifications d'identité (y compris l'identité sexuelle) mise en action par la pratique culturelle d'une homographèse-régulation qui trace les lignes de démarcation de l'espace au sein duquel penser l'« homosexualité » même. En somme, comme l'écriture, elle se dé(s-é)crit ellemême dans le temps même de son inscription.

18 L'utilité de cette implication homographique pour le concept d'homographèse deviendra peut-être plus claire si nous revenons à la question originelle de la définition de la «différence homosexuelle». Car la littérature dans laquelle l'homosexualité pénètre dans le champ de vision occidental arrive, de manière caractéristique, à ce qui passe pour un moment de révélation ou de reconnaissance sexuelle, mais, à y regarder de plus près, ce moment peut être vu comme le point auquel ce qui est « reconnu » est également constitué et produit, le point auquel un acte d'interprétation rétroactive s'exprime comme acte de "clarification» visuelle ou perceptive. Je prendrai, pour examiner le processus, deux brefs exemples choisis dans des textes canoniques qui engagent, chacun de manière différente, une graphèse de l'homosexualité.

19 Le premier exemple que je veux apporter est un moment de reconnaissance tiré du Portrait de Dorian Gray - ou plutôt, c'est la juxtaposition temporelle de deux moments séparés mais apparemment analogues du texte. Dans le premier, Dorian écoute la diatribe de Lord Henry Wotton contre les lenteurs et l'influence, et son appel au courage de réaliser sa propre identité, sa propre nature :

Mais l'homme le plus brave d'entre nous a peur de lui-même. Les mutilations que s'infligent les sauvages survivent tragiquement dans ce refus de nous-mêmes qui abîme nos vies. [...] La seule manière de se débarrasser d'une tentation est d'y succomber. Résistez-y, et votre âme tombe malade de la soif, des choses qu'elle s'interdit, du désir de ce que ses lois monstrueuses ont rendu monstrueux et illégal. [...] Vous, monsieur, oui, vous-même, avec votre jeunesse rose tirant sur le rouge et votre enfance blanche tirant sur le rose, vous avez eu des passions qui vous ont fait peur, des pensées qui vous ont terrifié, des rêves de jour ou de nuit dont le souvenir pourrait tacher de honte votre joue... (Wilde $2001: 60-61$ )

Bien que Dorian interrompe Lord Henry à ce moment-là, la logique sinueuse et insinuante de ces paroles produit une révélation qui l'éblouit : «Oui, il y avait eu des choses dans son enfance qu'il n'avait pas comprises. Il les comprenait maintenant. La vie soudain se para pour lui de toutes les couleurs du feu. Il lui sembla qu'il avait marché au milieu des flammes. Pourquoi ne s'en était-il pas rendu compte? » (61)

Si la vie de Dorian éclate en couleurs de feu juste au moment où il semble se rendre compte que, tout ce temps, « il avait marché au milieu des flammes », c'est parce que sa perception a été influencée par la construction tropologique, les couleurs rhétoriques si efficacement déployées dans les paroles de Lord Henry. Et puisque cette rhétorique insiste sur le besoin de « réaliser à la perfection notre propre nature » (59), elle s'inscrit dans l'idéologie d'identité et d'essence qui caractérise la métaphore. L'effet du discours séducteur de Lord Henry, qui oppose l'assujettissement à l'influence d'autrui - qui, selon son argument, fait de l'individu simplement l'acteur d'« un rôle qui n'a pas été écrit pour [lui] » (60) - se fait jour alors que Dorian devient « vaguement conscient des influences toutes nouvelles qui œuvraient en lui. Et pourtant, elles semblaient vraiment provenir de lui » (61). 
22 À un moment saturé d'ironie, le discours qui dénonce l'influence devient lui-même une puissante influence et l'idéologie de la totalisation métaphorique, de l'essence, implicite dans le discours de Lord Henry - l'idéologie qui insiste sur la nécessité de « réaliser à la perfection notre propre nature »-produit la « nature » ou sujet même qu'elle semble ne faire que révéler. Dorian semble admettre cela quand il laisse sa pensée vagabonder sur l'éloquence de Lord Henry juste avant le moment de son illumination soudaine :

Les mots! Rien que des mots! Mais qu'ils étaient donc redoutables! Qu'ils étaient clairs, vifs et cruels! On ne pouvait leur échapper. Et pourtant, ils contenaient une magie subtile! Ils semblaient capables de donner une forme plastique à des choses informes et de répandre une musique à eux [...]. Y a-t-il rien de plus réel que les mots? (61)

C'est bien sûr à Dorian lui-même que les paroles de Lord Henry ont donné « une forme plastique » en rendant possible cette révélation du sens de son expérience : de fait, leur pouvoir d'information peut être perçu tout particulièrement dans la manière dont elles permettent à Dorian de comprendre son état antérieur comme état d'enfance « informe » et totalement dénué de capacité d'intelligence.

Dans un essai intéressant sur Le Portrait de Dorian Gray, Ed Cohen fait remarquer le processus travers lequel, pour reprendre sa formulation, «le langage de Lord Henry crée une nouvelle réalité pour Dorian », si bien que «le concept que le jeune homme a de son propre être matériel s'en trouve modifié - il "est révélé à lui-même"15 " (Cohen 1987 : 808). Tandis que l'intérêt de Cohen s'attache surtout à la constitution de "l'être matériel » de Dorian ou à ce qu'il appelle ailleurs les « représentations de son identité » (Cohen 1987: 806), je m'intéresse plus à la manière dont l'identité finit par être un trope de la représentation-spécifiquement un trope de correspondance métaphorique qui affirme sa domination sur la contingence métonymique à laquelle elle s'accroche et qu'elle ranime en y injectant du sens.

Cohen juxtapose de manière tout à fait pertinente l'effet produit sur Dorian par les paroles de Lord Henry et l'effet produit sur le jeune homme par le portrait de Basil Hallward : «Dorian ne répondit pas. Il passa nonchalamment devant son portrait, puis se retourna. Quand il le vit, il se rejeta en arrière, et sa joue se colora un instant de plaisir. Une expression de joie surgit dans ses yeux, comme s'il s'était reconnu pour la première fois. » (67) Faisant implicitement entrer en jeu la théorisation du stade du miroir que Lacan a élaborée, Cohen commente ainsi la réaction de Dorian :

L'image organise les perceptions distinctes de son corps en un tout apparemment autonome et réoriente Dorian en relation à la fois à sa propre identité et à son contexte. [...] L'identification de Dorian avec l'image peinte constitue une fausse reconnaissance autant qu'une reconnaissance, ce qui le conduit à confondre un ensemble de représentations avec la « vérité » de son expérience. (Cohen 1987 : 808)

On pourrait repositionner cette interprétation lacanienne de cet instant d'autointerprétation chez Dorian en imaginant le personnage comme entretenant une relation homographique avec son image peinte - c'est-à-dire une relation d'identité apparente dans laquelle les signifiants sont perçus comme étant les mêmes ou comme se reflétant en miroir, même si le privilège, d'essence métaphorique, accordé à l'image, méconnaît la contingence, "l'accident" qui l'a produite. Après tout, comme Basil l'explique à Lord Henry, « tout portrait peint avec sincérité est le portrait de l'artiste et non du modèle. Le modèle n'est que l'accident, l'occasion. Ce n'est pas lui qui est révélé par le peintre ; c'est plutôt le peintre qui, sur sa toile peinte, se révèle lui-même » 
(Wilde 2001 : 46). De manière très révélatrice, quand Dorian se trouve si profondément affecté par les paroles de Lord Henry qu'elles "semblaient vraiment provenir de lui ", les paroles qui produisent cet effet de découverte de et sur soi et qui permettent en même temps à Basil de terminer son tableau en saisissant «l'effet qu['il] voulai[t]: les lèvres entrouvertes, la lumière dans les yeux » (62), sont explicitement situées dans le registre du hasard :

Les quelques mots que lui avait dits l'ami de Basil, des mots prononcés au hasard sans doute, et en cherchant délibérément le paradoxe, avaient effleuré en lui une corde secrète qui n'avait jamais été touchée plus tôt mais qui, il le sentait, vibrait et palpitait maintenant au rythme d'un pouls étrange. (61)

L'accident du discours de Lord Henry, avec son sujet choisi de manière arbitraire, produit la constitution de la subjectivité de Dorian par une identification métaphorique à une image particulière et l'appropriation de cette image - qu'elle soit littéralisée dans le portrait de Basil ou figurée dans la «forme plastique » que son expérience acquiert quand il entend les paroles de Lord Henry - paroles auxquelles sa relation serait décrite de manière plus précise en termes de contiguïté métonymique. Tout comme Dorian interprétera comme scène de reconnaissance le moment où il produit en fait ce qu'il (mé)prend pour sa propre identité en regardant le tableau auquel son rapport n'est jamais, selon les termes de Basil, qu' "accidentel» ou «occasionnel», de la même manière, il configure sa vie et lui donne la cohérence d'un récit formé par une nécessité interne en réaction aux paroles de Lord Henry, que le roman qualifie de «prononcées au hasard ». Ainsi, tandis que Lord Henry, dans son appel à "s'épanouir » comme le «but de la vie », fustige ceux qui acceptaient passivement d'être influencés, les tenant pour rien de mieux qu'un "écho de la musique d'un d'autre» (59), Dorian ne peut acquérir son identité que par l'influence de cette dénonciation de l'influence, puisque la « corde secrète » qu'il reconnaît faussement comme la réalisation de sa nature vibre d'une énergie qui ne provient pas de lui, une énergie transmise par l'effleurement, le hasard du contact qui caractérise la métonymie ${ }^{16}$.

Ironiquement, après cette cristallisation de son identité par le biais de la fausse reconnaissance de son homographe (figuré dans le tableau peint et le portrait verbal d'un moi autonome, donc après sa propre naturalisation métaphorique du contigu), Dorian se met en devoir de répudier précisément le registre d'identité métaphorique à travers lequel cette (mé)connaissance de soi a été produite. Car l'identité propre qu'il finit par se fixer est de celles qui refusent le concept d'identité fixée - comme Wilde le remarque à son propos plus loin dans le texte: "Il s'étonnait de la psychologie superficielle de ceux qui conçoivent le moi de l'homme comme simple, permanent, certain et composé d'une seule essence.» (193) Ainsi, pour avoir constitué métaphoriquement son identité à travers l'homographe, Dorian arrive à une lecture homographique de l'identité, une lecture de l'essence et du «moi» en termes de différences et de divisions rapprochés uniquement par un contact métonymique. Mais le refus de l'identité fixée est lui-même un effet de l'identification nécessairement instable de Dorian avec son image peinte - une identification posée par le biais (et à travers toute la gamme) des différences que le portrait ouvre au cœur de la notion d'« identité » en tant que telle. Naturellement, au premier plan de ces différences, on trouve la question de la différence sexuelle, une question qui a conduit à une curieuse ligne d'interrogatoire pendant le procès en diffamation du Marquis de Quinsberry. Dans son contre-interrogatoire de Wilde, Edward Carson, avocat de l'accusé, fit inscrire dans 
les minutes un passage de Dorian Gray dans lequel Basil avertit Dorian qu'on dit « les pires choses » de lui dans Londres :

Il ne faut pas que les gens parlent de toi comme d'un être vil et dégradé. Oui, tu as ta position, ta fortune et tout cela. Mais la position et la fortune ne sont pas tout. Remarque que, ces rumeurs, je n'y crois pas. Ou du moins, je n'y crois pas quand je te vois. Le péché s'inscrit sur le visage de l'homme. On ne peut pas le dissimuler. Les gens parlent quelquefois de vices cachés. Cela n'existe pas. Si un malheureux a un vice, il se montre dans les plis de sa bouche, la chute de ses paupières, même la forme de ses mains ${ }^{17}$. (Wilde $2001: 200$ )

Laissant de côté ce qui lui semblait n'être qu'une série élaborée d'échappatoires, Carson poursuit en demandant carrément et en s'en tenant à une lecture littérale (mais non erronée pour autant) : «Ce passage ne suggère-t-il pas une accusation de vice contrenature $^{18}$ ?» (Hyde 1956: 132) Même si Wilde évite de répondre directement à la question, sa manière de déployer le trope de la lisibilité dans le passage cité par Carson attire l'attention sur un trait de son roman qui pourrait bien avoir contribué à l'impression troublante qu'il a produite sur les lecteurs de l'époque : le lien évident de Dorian à un monde de "vice contre-nature » ne produit pas l'inscription " appropriée » de la différence sur son corps; au lieu de cela, cette inscription est déplacée sur le tableau avec lequel il entretient d'abord une relation faussement reconnue comme une similarité allant de soi, comme identité métaphorique. Cette similitude ou identité est à la fois renforcée et subvertie au fur et à mesure que le portrait seul, « le plus magique des miroirs » (154) se couvre des marques de la différence inscrites par les actes illicites de Dorian, le transformant en « emblème visible de la conscience » (139) et le « symbole visible de la dégradation du péché » (143). De la même manière que la ressemblance initiale du portrait cachait sa dissemblance homographique, de la même manière, Dorian lui-même - comme le roman dans lequel il figure - menace, comme incarnation de la différence sexuelle indifférenciée, de réduire à néant l'assurance avec laquelle la ressemblance de l'identité (hétérosexuelle) peut être reconnue. Même la conclusion moralisatrice du roman, dans laquelle les attributs propres du portrait et de la personne sont restaurés de manière rassurante et la certitude d'Hallward que " [1]e péché s'inscrit sur le visage de l'homme » est validée par l'image du cadavre de Dorian gisant «flétri, ridé, son visage [...] répugnant » (277), même cela ne peut compenser complètement la possibilité troublante soulevée par le texte que la ressemblance sur laquelle l'identité s'appuie se révèle n'être qu'un homographe qui masque une différence d'une lisibilité aussi déroutante et aussi perturbante pour l'ordre de la logique sociale, que celle qui existe entre Dorian Gray et le portrait qui représente sa (fausse) reconnaissance de soi.

Je voudrais recontextualiser brièvement les questions de reconnaissance et d'inscription abordées dans Le Portrait de Dorian Gray en introduisant comme second exemple de fausse reconnaissance homographique un passage tiré de l'ouverture de Sodome et Gomorrhe de Proust, texte qui concentre la plus grande partie de son énergie sur la visualisation de l'homosexuel, littéralisant ce but dans l'observation par le Narrateur du jeu de séduction entre M. Jupien et le Baron de Charlus. Dans un langage qui rappelle le moment où Dorian accède à la reconnaissance, le narrateur, après avoir observé le rituel du désir pratiqué par ces deux hommes, déclare : "Dès le début de cette scène une révolution, pour mes yeux dessillés, s'était opérée en $M$. de Charlus, aussi complète, aussi immédiate que s'il avait été touché par une baguette magique. 
Jusque-là, parce que je n'avais pas compris, je n'avais pas vu. » (Proust 1988 : 15) À ce moment-là, c'est Charlus qui, nous dit le texte, subit une transformation, mais cette

Affirmation déplace en fait la transformation qui affecte le narrateur lui-même à mesure qu'il découvre, en observant la scène, le double impératif d'une lecture homographique - en d'autres termes, à mesure qu'il apprend, non seulement que l'apparence de similitude peut cacher une différence (de "sens ", si l'on peut dire) désorientante au cœur même de chacune des identités sexuées à travers lesquelles le symbolique articule les sujets, mais aussi qu'une attention disciplinée peut récupérer la cohérence idéologique de l'identité précisément par le biais de la vigilance avec laquelle elle cherche et « lit » la catégorie de personne construite de manière projective pour incarner cette rupture déstabilisante de l'identité elle-même, et produire du sens en l'assumant comme identité caractérisante. Il est significatif à cet égard que le passage retourne le processus normal de production du sens qui permet à l'épistémologie occidentale de représenter sa rationalité ou sa logique comme allant de soi. La vision ne précède plus, pour produire, comme par « nature », la compréhension. Celle-ci devient le prérequis pour un acte ultérieur de vision, apparu comme sous l'effet d'un coup de baguette magique qui figure le rôle de la perception idéologique comme agent transformateur. Autrement dit, en accord avec la mission disciplinaire de l'homographèse, l'élaboration conceptuelle d'une différence sexuelle binaire hétéro/ homo engendre, à titre d'analogie, une inscription différée de l'«identité » sexuelle dans le champ du visuel.

31 Comme c'est le cas lorsque Dorian entend les paroles de Lord Henry, ou lorsqu'il voit le tableau de Basil pour la première fois, ce moment de reconnaissance produit, de manière rétroactive, les "significations" variées qu'il paraît au contraire révéler. Ainsi, la « vérité » de la sexualité de Charlus, lorsqu'elle est lue comme la métaphore ou l'essence de son identité, investit de sens les actions qui avaient jusque-là été comprises comme purement contingentes; mais cette lecture de Charlus comme homographe comme signifiant dont l'identité à soi apparente représente faussement une différence qui peut passer inaperçue, sans être pourtant imperceptible - démantèle l'intégrité de l'identité de Charlus pour refigurer aussitôt la différence, la contradiction interne qui la déconstruit comme graphèse de son identité catégorique d'homosexuel. Et, dans le passage sur lequel je veux conclure ces remarques extrêmement schématiques à propos de Proust, le narrateur exprime cette homographèse en des termes qui situent la problématique de la visibilité gay explicitement en relation avec les problématiques de l'écriture ou graphèse :

En M. de Charlus un autre être avait beau s'accoupler, qui le différenciait des autres hommes, comme dans le centaure le cheval, cet être avait beau faire corps avec le baron, je ne l'avais jamais aperçu. Maintenant l'abstrait s'était matérialisé, l'être enfin compris avait aussitôt perdu son pouvoir de rester invisible, et la transmutation de $\mathrm{M}$. de Charlus en une personne nouvelle était si complète que non seulement les contrastes de son visage, de sa voix, mais rétrospectivement les hauts et les bas eux-mêmes de ses relations avec moi, tout ce qui avait paru jusque-là incohérent à mon esprit, devenait intelligible, se montrait évident, comme une phrase, n'offrant aucun sens tant qu'elle reste décomposée en lettres disposées au hasard, exprime, si les caractères se trouvent replacés dans l'ordre qu'il faut, une pensée que l'on ne pourra plus oublier. (Proust $1988: 16$ )

32 Nous avons ici affaire au déploiement de l'homographèse parfaitement au fait de l'acte rétrospectif d'interprétation qui produit un sens à partir de phénomènes d'abord compris comme arbitraires et sans conséquences. Le modèle de cette homographèse est 
emprunté explicitement à la lisibilité de l'écriture même - à "l'ordre qu'il faut ", qui nous permet de donner sens à ce qui, autrement, se présente comme inintelligible comme autant de «lettres disposées au hasard». L'attribution de propriété et de nécessité à cette mise en ordre, sa manière de dévoiler le «sens" par une mise en perspective, invoque la logique dominante de la métaphore comme figure de la présence du «sens" en tant que tel, mais le "sens » qui devient «évident en soi ", à travers l'instauration de l'ordre approprié entre ces lettres aléatoires, reste enraciné dans la contiguïté métonymique des graphèmes par lesquels la phrase qu'ils forment prend forme. Ainsi, cette métaphore de la lisibilité de Charlus comme homosexuel, cette métaphore de la graphèse de l'homosexualité elle-même, signale le caractère signifiant d'une identité propre - c'est-à-dire métaphorique - qui ne peut être produite qu'à travers la relation syntagmatique caractéristique de la métonymie.

\section{Homographèse : pour une pratique critique et politique gay}

33 Comment, pourrait-on ici demander, ce genre d'analyses rhétoriques des figurations de la lisibilité homosexuelle peut-il jeter la moindre lumière sur la question initiale de l'émergence d'une pratique critique gay? À quelles interventions le concept d'homographèse peut-il conduire dans le champ de la politique de la sexualité ? En premier lieu, ma réponse serait qu'une reconnaissance de l'inscription du corps gay comme écriture ou comme texte suggère qu'il faut compter parmi les projets nécessaires pour les critiques gay et pour le champ de la théorie gay, en pleine extension, l'étude de la rhétorique historiquement variable, des stratégies textuelles et des formations tropologiques dans lesquelles la sexualité est enchâssée et conçue ; elle suggère que les diverses psychologies de la figuration, dans divers lieux et à diverses époques, ont des répercussions cruciales sur les articulations textuelles et les constructions culturelles de la sexualité; et elle suggère aussi que la sphère d'une critique gay ne doit pas forcément se limiter à l'examen de textes qui, ou bien thématisent les relations homosexuelles, ou bien dramatisent les vicissitudes du désir homosexuel/homosocial. En se focalisant sur l'émergence historique de l'homosexualité dans un rapport rigide à des questions de pouvoir social et à la constitution d'identité, le projet de l'homographèse situerait la force critique de l'homosexualité précisément au point de discrimination entre ressemblance et différence, comme repères cognitifs gouvernant le champ discursif des relations symboliques sociales. Non seulement la logique de l'identité sexuelle, mais celle qui informe la tropologie par le biais de laquelle l'identité et la différence elles-mêmes sont construites, enregistrées et mises en œuvre par l'opération naturalisée de la Loi, devient par là susceptible d'une analyse critique gay.

Mais je n'ai pas plus tôt avancé ces hypothèses que je m'avise d'une critique que le paragraphe précédent peut susciter : car faire de la relation entre la rhétorique et la tropologie, d'une part, et les mécanismes psychiques de l'identité et du désir, d'autre part, le centre des préoccupations de la théorie gay, risque d'attirer l'accusation de sembler défendre activement ou du moins tolérer un formalisme apolitique. Or, c'est justement la politique incontournable de tout formalisme, l'insistance de l'idéologie dans toute graphèse de la sexualité (gay) quelle qu'elle soit (pour autant qu'elle cherche à articuler et à réifier la forme même, la morphologie, comme structure signifiante de 
l'identité), que l'étude de l'homographèse prend comme point de départ. Procéder autrement, rester prisonnier de l'enchantement d'un fantôme d'engagement politique en dehors et au-dessus de l'engagement dans les problématiques de la rhétorique, de la figuration et du fantasme, c'est ignorer que l'homosexualité a été conçue dans l'histoire dans un rapport distinctif au langage et avaliser une manière de comprendre l'interprétation qui, comme Paul de Man le dit dans un autre contexte, constitue

le vivier privilégié de modèles fallacieux et de métaphores trompeuses. Cette conception de l'interprétation est responsable du modèle métaphorique de la littérature comme une sorte de boîte qui sépare un dedans d'un dehors, et du lecteur ou du critique comme une personne qui soulève le couvercle pour faire sortir au grand jour ce qui était dissimulé, mais inaccessible à l'intérieur. (de Man 1979:5)

Comme ces formulations l'impliquent, le privilège métaphysique de la métaphore et de sa logique essentialisante peut être vu comme le "vivier» dans lequel un ordre hétérosexuel (re)produit l'idéologie de l'identité en articulant de manière prescriptive une relation hiérarchique entre des catégories définies comme des pôles opposés. La valence hétérosexuelle de cette métaphore est particulièrement évocatrice dans le passage cité ci-dessus parce que ce "vivier de modèles fallacieux et de métaphores trompeuses » engendre un paradigme selon lequel lire, ou interpréter, revient à ouvrir une boîte pour révéler une vérité qui était «dissimulée, mais inaccessible à l'intérieur ». S'il est difficile, dans le contexte de cet essai, de ne pas faire de cette boîte qui contient un secret une variante du placard (et ce, d'autant plus que de Man éclaire sa remarque par une référence à un passage de Du côté de chez Swann de Proust dans lequel, pour reprendre la glose suggestive de de Man, la grand-mère du narrateur le pousse à "quitter l'intérieur malsain de son cabinet de lecture »), il est également difficile de ne pas y voir une figure du corps gay comme homographe, imaginé avec angoisse comme contenant une « différence » qui menace de rester « dissimulée, mais inaccessible à l'intérieur ». La discipline culturelle de l'homographèse comme pratique qui vise à la « libération » ou révélation de cette "vérité » qu'est l'identité par le biais d'un "modèle métaphorique " de la lecture, réagit de manière défensive à cette menace, et, ce faisant, donne une idée de la structure hétérosexuelle implicite qui informe la croyance que le privilège de l'interprétation est intrinsèquement lié à «la métaphore "intérieur/extérieur" "(De Man 1979:5).

Mais c'est cette métaphore intérieur/extérieur qui fait que les homophobes aussi bien que les anti-homophobes insistent sur la distinction entre straight et gay. Une telle institutionnalisation de la différence, selon mon raisonnement, a pour effet de reconfirmer la logique de l'identité, la ressemblance du moi ; en affirmant la lisibilité de la différence sexuelle, les sujets sociaux, straight ou gay, accèdent à un instrument puissant pour constituer et mobiliser les énergies «de la communauté ». Mais l'enjeu n'est pas le même pour les lecteurs straight et les lecteurs gay qui insistent sur la lecture (qui est aussi une inscription) de la différence sexuelle. Plutôt que de réinscrire dans notre pratique critique ce modèle de la discrimination sexuelle intérieur/ extérieur, ou/ou, déterminé par l'hétérosexualité, nous serions bien inspiré/es, c'est-àdire les critiques gay et lesbiennes seraient bien inspirés de prendre en considération la description que Barbara Johnson fait d'une critique déconstructrice qui viserait "à développer un discours qui ne dit ni "ou/ou", ni "et/et", ni même "ni/ni", sans pour autant abandonner non plus totalement ces logiques" (Johnson 1987 : 12). Car, même si les conceptualisations métaphoriques de la sexualité ont pu servir tel ou tel groupe, 
telle ou telle occasion, et même si la disruption métonymique des "identités sexuelles » peut sembler tentante, nous devons garder à l'esprit, comme Jane Gallop l'écrit à propos de la lecture du genre sexué [gender], que "toute opposition polaire entre métaphore et métonymie (vertical vs horizontal, masculin vs féminin) est enfermée dans l'ordre imaginaire, soumise au jeu de l'identification et de la rivalité " (Gallop 1985 : 132) - en d'autres termes, qu'elle reproduit le binarisme qui sous-tend la logique de l'identité et qui informe le "modèle métaphorique» de la lecture. Les fausses reconnaissances par lesquelles l'antithèse hétéro / homo modèle notre monde exigent une analyse rigoureuse, sophistiquée sur le plan rhétorique et attentive à la psychanalyse, si l'on veut justement imaginer une politique capable de prendre en compte les complexités d'un sujet qui ne peut parler que du cœur de ces fausses reconnaissances dans l'étau desquels il est pris.

L'analyse rhétorique dont on a besoin dans la pratique stratégique de la de-scription refuse donc de se reconnaître comme différente de ce qui est politiquement engagé. C'est-à-dire qu'elle refuse l'organisation rhétorique du politique lui-même ${ }^{19}$. Le positionnement historiquement déterminé de l'homosexualité à l'intersection ambiguë du métaphorique et du métonymique peut aider à rendre compte de phénomènes actuels comme l'insistance brutale sur une identité homosexuelle spécifique et lisible qui sous-tend la fréquence et la violence croissantes des agressions sur les gays, et il peut éclairer la persistance de la croyance démentie par les faits en une dissémination du sida par contagion métonymique. Ce ne sera pas assez de rendre compte de phénomènes de ce genre pour y mettre fin, j'en conviens, mais cela peut nous permettre de formuler des stratégies au moyen desquelles faire face de manière plus efficace aux impératifs du moment, et cela peut nous aider à voir comment certains actes de résistance peuvent eux-mêmes être empêtrés dans les logiques sous-jacentes et reproduire ainsi précisément les structures qui aboutissent à notre oppression.

Si le projet d'une homographèse déconstructrice ne peut jamais réussir à se dégager complètement de l'homographèse régulatrice contre laquelle elle voudrait trouver un moyen de pression, cela ne fait que témoigner de l'émergence de la théorie gay du sein du discours symbolique qui exige la réification des identités. Écrire sur la discipline culturelle qui articule l'homosexualité en référence à l'écriture, c'est produire un autre moment dans le même champ discursif; mais la démarche d'une homographèse oppositionnelle stratégique vise à faire une différence politique en s'attaquant aux implications idéologiques du marquage de la différence sexuelle. Car pour échapper à la fois aux constructions d'une sexualité réduite au silence et aux dangers d'une sexualité inscrite comme essentielle, nous devons construire de manière rétroactive, à partir des accidents divers qui constituent l'histoire, une différence à la logique hétérosexuelle de l'identité - appuyée qu'elle est aujourd'hui par la notion d'une différence sexuelle reniée et projetée - pour déconstruire l'idéologie répressive de la similitude ou de l'identité elle-même. 


\section{BIBLIOGRAPHIE}

Baldwin, J., 1964, Un autre pays, J. Autret (trad.), Paris, Gallimard, coll. « NRF ».

Blackstone, Sir W., 1899, Commentaries on the Laws of England, J. DeWitt Andrews (éd.), vol. 2, Chicago.

Bray, A., 1982, Homosexuality in Renaissance England, Londres, GMP.

Burger, W., 1986, « The Supreme Court Opinion: Michael J. Bowers, Attorney General of Georgia, Petition v. Michael Hardwick and John and Mary Doe, Respondents ", New York Native, July 14

Cleland, J., 1985, Memoirs of a Woman of Pleasure, P. Sabor (éd.), New York, Oxford University Press. Cohen, Ed., 1987, « Writing Gone Wile. Homoerotic Desire in the Closet of Representation », PMLA, no 102 (Autumn 1987), p. 801-813.

De Man, P., 1979, « Semiology and Rhetoric », Allegories of Reading. Figurai Language in Rousseau, Nietzsche, Rilke, and Proust, New Haven, Yale University Press, p. 3-19.

Derrida, J., 1967, De la grammatologie, Paris, Minuit.

-, 1979, L'Écriture et la Différence, Paris, Le Seuil.

Edelman, L., 1994, Homographesis: Essays in Gay Literary and Cultural Theory, New York, Routledge.

Foucault, M., 1976, Histoire de la sexualité. I. La Volonté de savoir, Paris, Gallimard.

Hyde, H. M. (éd.), 1956, The Three Trials of Oscar Wilde, New York, University Books.

Johnson, B., 1987, A World of Difference, Baltimore, The Johns Hopkins University Press.

Gallop, J., 1985, Reading Lacan, Ithaca, Cornell University Press.

Karlen, A., 1971, Sexuality and Homosexuality: A New View, New York, Norton.

Lacan, J., 1973a, « La sexualité dans les défilés du signifiant », dans Les Quatre Concepts fondamentaux de la psychanalyse, Le Séminaire XI, Paris, Le Seuil, p. 167-180.

- 1973b, « La pulsion partielle et son circuit », Les Quatre Concepts fondamentaux de la psychanalyse. Le Séminaire XI, Paris, Le Seuil, p. 195-209.

Laplanche, J., 2008 [1970], Vie et Mort en psychanalyse, Paris, PUF.

Logan, M.-R., 1975, « Graphesis... », dans M.-R. Logan (dir.), Graphesis: Perspective in Literature and Philosophy, Yale French Studies, ${ }^{\circ}$ 52, p. 4-15.

Proust, M., 1988, À la recherche du temps perdu, Paris, Gallimard, coll. « Bibliothèque de la Pléiade », sous la dir. de J.-Y. Tadié, vol. 3.

Radzinowicz, Sir L., 1968, A History of the English Criminal Law, vol. 4, Grapplingfor Control, Londres, Stevens and sons.

Sedgwick, E. K., 1985, Between Men: English Literature and Male Homosocial Desire, New York, Columbia University Press.

-, 1986, «Comments on Swann », Berkshire Review, $\mathrm{n}^{\circ}$ 1, p. 107-113.

-, 1988, « The Epistemology of the Closet (I) », Raritan, $n^{\circ} 7,4$ (Spring 1988), p. 39-69. Textes repris dans The Epistemology of the Closet, Berkeley et Los Angeles, The University of California Press, 1990. 
Trumbach, R., 1977, « London's Sodomites: Homosexual Behavior and Western Culture in the Eighteenth Century », Journal of Social History I, $n^{\circ}$ 11, 1 (Fall 1977), p. 1-13.

- 1987, « Sodomitical Subcultures, Sodomitical Roles and the Gender Révolution of the Eighteenth Century : The Recent Historiography », dans R. Purks MacCubbin (dir.), 'Tis Natures Fault: Unauthorized Sexuality during the Enlightement, New York et Cambridge, Cambridge University Press, p. 109-121.

Weeks, J., 1981, « The Construction of Homosexuality », dans Sex, Politics and Society: The Regulation of Sexuality since 1800, New York, Longman.

Wilde, O., 1996 [attribué à], Teleny. Étude physiologique, Paris, Le Pré-aux-clercs.

-, 2001, Le Portrait de Dorian Gray, V. Volkoff (trad.), Paris, Librairie Générale Française, coll. « Livre de poche classique ».

\section{NOTES}

2. Le "placard» est l'équivalent en français de "closet» en anglais, qui concrétise le secret imposé socialement aux homosexuels qui, lorsqu'ils rendent publique leur "orientation sexuelle » sortent du placard (à moins qu'un geste public, le outing, n'expulse un individu en général haut placé, mais considéré comme de mauvaise foi) de la sécurité relative de cet enfermement. Le terme a acquis une valeur théorique qui double les enjeux politiques, en particulier avec le livre d'Eve Kosofsky Sedgwick, The Epistemology of the Closet (1990) qui reprend un essai du même titre, publié en 1988. Le livre vient d'être traduit en français, sous le titre Épistémologie du placard (Paris, Éditions Amsterdam, 2008) [NDT].

3. Sauf indication contraire, toutes les traductions des textes cités sont dues au traducteur de l'article.

4. "Whatever effect this infamous passion had in other ages, and other countries, it seem'd a peculiar blessing on our air and climate, that there was a plague-spot visibly imprinted on all that are tainted with it, in this nation at least. » (Cleland $1985: 159$ )

5. Trumbach arrive aussi à la même conclusion : «Je rejoindrais maintenant Mary McIntosh pour penser qu'un changement profond est intervenu dans la conceptualisation et la pratique des comportements homosexuels masculins à la fin $\mathrm{du}^{\mathrm{xVII}}{ }^{\mathrm{e}}$ et au début du xvIII ${ }^{\mathrm{e}}$ siècle. Ce changement a été provoqué par la réorganisation de l'identité des genres sexuée, qui constituait un élément de l'émergence d'une culture occidentale. » (Trumbach $1988: 118$ )

6. «Epistemology of the Closet (I) » a été revu et repris dans Sedgwick 1990 (Epistemology of the Closet). Une présentation antérieure de l'argumentation figure dans Sedgwick 1985.

7. L'expression originale, « it takes one to know one » n'a guère d'équivalent français (NDT).

8. Voir Lacan 1973a.

9. Cité dans Gallop 1985 : 124. [Le texte cité en anglais est le suivant : «metonymy is there from the beginning and is what makes metaphor possible.» (NDT)] Nous pouvons réinterpréter les paroles de Lacan pour suggérer que la métaphore impose du sens à une relation métonymique antérieure qui ne peut être reconnue comme sens que du fait qu'elle est lue comme métaphorique. Ce modèle rappellera bien sûr le processus du sens différé ou rétroactif dont l'opération est pour Freud cruciale dans la constitution de la sexualité elle-même. Voici la manière dont Laplanche articule le lien intime entre sexualité et ses différés : «[P]ourquoi la sexualité ? La réponse de Freud c'est que seule la sexualité est susceptible de se prêter à cette action en deux temps qui est aussi une action après coup. C'est là et seulement là que nous trouverions ce jeu complexe et sans cesse répété, au sein d'une succession temporelle faite d'occasions manquées, de "trop tôt" et de "trop tard” " (Laplanche 2008 : 71). Les réflexions ci-dessous donneront, je l'espère, une importance 
rétroactive à cette notion de sens rétroactif. Notons déjà la formulation de Lacan : «La lisibilité du sexe dans cette interprétation des mécanismes inconscients est toujours rétroactive.» (Lacan 1973 b : 198)

10. Le présent article est exclusivement consacré à des problématiques liées à l'homosexualité masculine, non pas parce que les problématiques liées à l'inscription lesbienne sont sans intérêt, mais parce que les problématiques impliquées sont à mon avis constituées de manière très différente. Bien que le lesbianisme, une fois qu'il parvient à être articulé publiquement, soit lu avec la grille de l'homosexualité masculine, cette lecture est, elle-même, une appropriation masculiniste qui a une histoire et une sociologie propres. Même si les lesbiennes et les gays ont été, et restent, la plupart du temps, des alliés dans la lutte pour la reconnaissance de leurs droits civiques, le fait d'être engagés dans des relations homoérotiques, qui leur est commun, ne doit pas masquer les différences dans l'expérience vécue qui résultent des différences dans le positionnement social au sein d'une culture qui répartit les êtres humains entre catégories distinctes - le masculin et le féminin.

11. Voir sa discussion de l'écriture dans «Ellipse »: « Dès qu'un signe surgit, il commence par se répéter. Sans cela, il ne serait pas signe, il ne serait pas ce qu'il est, c'est-à-dire cette non-identité à soi qui renvoie régulièrement au même. C'est-à-dire à un autre signe qui lui-même naîtra de se diviser. Le graphème, à se répéter ainsi, n'a donc ni lieu ni centre naturels. » (Derrida 1967 : 297)

12. Voir Trumbach 1977, en particulier p. 117. Voir aussi la description que fait en 1709 Ned Ward du comportement des homosexuels masculins qui se retrouvent dans les molly houses (lieux de rencontre - tavernes ou habitations privées - pour les homosexuels, les mollies, à la fin du XVII siècle et au siècle suivant): "Ils se prennent pour des femmes [...], affectant de parler, de marcher, de faire des commérages, de faire la révérence, de pleurer, de gronder, et d'imiter toutes les manières de l'efféminement » (cité par Trumbach 1977 : 12-13). Cleland fait dire à Mrs. Cole, dans Memoirs of a Woman of Pleasure, à propos des sodomites : «Ils étaient à peine moins détestables que ridicules dans leur incohérence monstrueuse, avec leur haine et leur mépris pour les femmes, alors qu'ils singeaient en même temps leurs allures, leurs façons de parler, de marcher, et, de manière générale toutes leurs petites affectations qui au moins leur vont mieux qu'à ces hommes-demoiselles qui ont perdu leur sexe. » (Cleland $1985: 160$ ) Cette insistance sur l'incohérence et la substitution parodique devraient rappeler les arguments traditionnels contre l'écriture que Derrida parcourt dans De la grammatologie: "L'inversion des rapports naturels aurait ainsi engendré le culte pervers de la lettre-image : péché d'idolâtrie, "superstition pour la lettre", dit Saussure dans les Anagrammes, où il a d'ailleurs du mal à prouver l'existence d'un "phonème antérieur à toute écriture". La perversion de l'artifice engendre des monstres. L'écriture comme tous les langages artificiels qu'on voudrait fixer et soustraire à l'histoire vivante de la langue naturelle, participe de la monstruosité. » (Derrida 1967 : 57)

13. Et le terme anglais lead est lui-même un homographe (le verbe conduire, avec le nom correspondant pour la position de tête, et le nom qui désigne le plomb).

14. Voir, par exemple, la remarque de Paul de Man, pour qui « l'inférence d'identité et de totalité est constitutive de la métaphore », dans « Semiology and Rhetoric », De Man 1979 : 14.

15. Sauf indication contraire, les citations sont traduites par Pierre Zoberman.

16. À ce stade, on pourrait penser aux discussions du développement du moi chez Freud - et spécialement dans leur reformulation par Jean Laplanche (Laplanche 1970). Comme Laplanche le suggère, le moi est une organisation qui lit son propre rapport métonymique à l'organisme vivant en termes métaphoriques, si bien qu'il en vient à désigner non seulement la partie, mais le tout, à travers cet acte de fausse reconnaissance.

17. Le texte anglais fait ici référence aux Three Trials of Oscar Wilde (Hyde 1956 : 131) [NDT].

18. Il vaut la peine de remarquer que la différence métaphoriquement appropriée comme identité par Dorian lorsqu'il se « reconnaît » est informée par celle de l'artiste, Hallward, dont l'identité sexuelle est inscrite dans l'œuvre d'art. En ce sens, Dorian Gray articule la circulation du 
désir homosexuel dans la notion d'«influence ». De manière significative, cette notion a aussi joué un rôle important dans les procès de Wilde. Niant que le passage cité ci-dessus suggère le "vice contre-nature ", Wilde déclare qu'il « décrit Dorian comme un homme d'influence très corrompu, bien qu'il n'y ait aucune déclaration sur la nature de cette influence ». Quelques instants plus tard, niant qu'un homme pût jamais corrompre un jeune homme, Wilde affirme : «Je ne pense pas qu'une personne puisse en influencer une autre. ( Hyde 1956: 132) Mais quand Wilde lui-même passa en justice, Alfred Wood témoigna contre lui, assurant que Wilde et lui « étaient montés dans une chambre où [ils] avaient bu du vin blanc à l'eau de Seltz. S'était alors produit un acte de la plus grande indécence. Mr. Wilde avait utilisé son influence pour obtenir mon consentement » (Hyde 1956 : 202).

19. Pour une analyse plus approfondie de cette question voir Edelman 1994, ch. 5, «The Mirror and the Tank : "AIDS," Subjectivity, and the Rhetoric of Activism » (93-117).

\section{RÉSUMÉS}

En inventant le terme " homographèse " pour nommer l'assemblage du corps homosexuel aussi bien avec la différence d'inscription graphique qu'avec l'illisibilité qui vient de son statut de «similitude» potentielle, cet article étudie les contradictions qui s'ensuivent quand le corps accède à la visibilité en tant que texte d'identité sexuelle, contradictions qui deviennent en ellesmêmes visibles quand la logique de la textualité remet en question les revendications identitaires. Cet essai situe le corps homosexuel au centre d'un combat pour la lisibilité corporelle dans lequel les forces aussi bien disciplinaires que contestataires ont intérêt à savoir « lire» ce corps comme un indice d'«identité sexuelle». De ce point de vue, l'essai examine la relation entre l'émergence d'une identité homosexuelle socialement articulable et sa connexion figurative à une idéologie culturellement suspecte de l'écriture.

Coining the term "homographesis" to name the conflation of the homosexual body both with the difference of graphic inscription and with the illegibility that arises from its status as potentially "the same," this essay explores the contradictions that follow when the body enters visibility as a text of sexual identity-contradictions that themselves become visible when the logic of textuality challenges identitarian claims. The essay situates the homosexual body at the center of a struggle over corporeal legibility in which both disciplinary and contestatory forces have a stake in being able to "read" that body as an index of a "sexual identity." From that perspective, the essay examines the connection between the emergence of a socially articulable homosexual identity and its figural connection to a culturally suspect ideology of writing.

\section{INDEX}

Mots-clés : identité sexuelle, homographe, genre, métaphore/métonymie

Keywords : sexual identity, homograph, gender, metaphor/metonymy 
AUTEURS

LEE EDELMAN

Tufts University 